\title{
Clinicopathological and Prognostic Significance of Epithelial Gremlin1 Expression in Gastric Cancer
}

\author{
RIRINO HONMA ${ }^{1}$, NAOYA SAKAMOTO ${ }^{1}$, AKIRA ISHIKAWA ${ }^{1}$, DAIKI TANIYAMA ${ }^{1}$, \\ KAHO FUKADA ${ }^{1}$, TAKUYA HATTORI ${ }^{1}$, KAZUHIRO SENTANI $^{1}$, NAOHIDE OUE ${ }^{1}$, \\ KAZUAKI TANABE $^{2}$, HIDEKI OHDAN ${ }^{2}$ and WATARU YASUI ${ }^{1}$ \\ ${ }^{1}$ Department of Molecular Pathology, Graduate School of Biomedical and Health Sciences, \\ Hiroshima University, Hiroshima, Japan; \\ ${ }^{2}$ Department of Gastroenterological and Transplant Surgery, Applied Life Sciences, \\ Graduate School of Biomedical \& Health Sciences, Hiroshima University, Hiroshima, Japan
}

\begin{abstract}
Background/Aim: Gastric cancer (GC) is one of the most common malignancies worldwide. Gremlinl is an antagonist of bone morphogenetic proteins that plays a critical role in several biological processes including cancer biology. Materials and Methods: We immunohistochemically examined the expression and distribution of Gremlin1 in non-neoplastic gastric mucosa in a series of 159 GC cases. Results: Among 159 GC primary tumors, 59 (37\%) were positive for Gremlin1. Gremlin1-negative GC cases showed significantly more advanced clinicopathologic factors and a trend toward intestinal-type GC. Gremlin1 expression was also frequently observed in MUC5AC-positive and G-type GC cases. Gremlin1-negative GCs had a poorer survival rate than Gremlin1-positive GCs ( $p=0.002)$. Univariate and multivariate analyses revealed that Gremlin1 expression is an independent predictor of survival in GCs. Conclusion: These results indicate that Gremlin1 could be involved in GC progression and may be a good marker of long-term survival in GC.
\end{abstract}

Gastric cancer (GC) is the fifth leading cause of cancerrelated death worldwide and the most frequent cancer in East Asian countries. A deeper understanding of the pathogenesis and biological features of GC is necessary to further inform and enhance early detection and treatment methods. One of the main strategies for the improvement of GC diagnosis and treatment is the identification of novel biomarkers that could be used to develop new tools for diagnosis and targets for

Correspondence to: Wataru Yasui, Department of Molecular Pathology, Graduate School of Biomedical and Health Sciences, Hiroshima University, 1-2-3, Kasumi, Minami-ku, Hiroshima, Japan, 734-8551. Tel: +81 822575145, Fax: +81 822575149, e-mail: wyasui@hiroshima-u.ac.jp

Key Words: Gremlin1, gastric cancer, prognostic factor. treatment (1). While we have identified several GC-specific genes (2), definitive biomarkers for accurately detecting GC and/or predicting the survival of GC patients are still lacking.

Gremlin1, a product of GREM1, is a bone morphogenetic protein (BMP) antagonist against several classes of BMPs (3). BMPs are well known as part of the TGF- $\beta$ superfamily and play an important regulatory role during organogenesis in the embryonic phase (4). In normal colon crypts, GREM1 is expressed by the intestinal peri-crypt fibroblasts and smooth muscle cells of the muscular mucosa, creating an increasing gradient toward the crypt. This gradient contributes to maintaining the intestinal stem cell niche in the colonic basal crypt region (5), which indicates that GREMI expression may influence the progression of colorectal cancer. Gremlin 1 is reportedly expressed in the myofibroblasts and smooth muscle cells at the bottom of crypts (5), as well as the crypt epithelium (6), and has been shown to be overexpressed in the epithelial cells of sporadic colorectal traditional serrated adenomas and in CRC $(7,8)$. Although one study has investigated the prognostic value of Gremlin1 expression in CRC cells (9), no studies have described the clinical significance of GREM1/Gremlin1 expression in GC. The present study evaluated the expression and role of Gremlin1 in GC by analyzing its expression and distribution using immunohistochemistry and examining the relationship between Gremlin1 positivity and clinicopathological features, including the prognostic significance. We also assessed the correlation between Gremlin1 expression and mucin phenotypes, which could provide evidence of a correlation between Gremlin1 expression and genetic background in GC.

\section{Materials and Methods}

Patients and tissues. Samples from 159 primary tumors were collected from patients diagnosed with GC who underwent surgery between 2005 and 2008 at Hiroshima University Hospital (Hiroshima, Japan). All patient samples were obtained with consent; 
this study was approved by the Ethics Committee for Human Genome Research of Hiroshima University (Hiroshima, Japan) and conformed to the ethical guidelines of the Declaration of Helsinki.

For immunohistochemical analysis, archival formalin-fixed, paraffin-embedded tissues from 159 patients who had undergone surgical excision for GC at Hiroshima University Hospital were consecutively collected. One or two representative tumor blocks from each patient, including the tumor center and tumor-associated non-neoplastic mucosa, were examined using immunohistochemistry. Tumor staging was determined according to the TNM classification system. Histological classification was carried out according to the Lauren classification (10).

Immunohistochemistry. Immunostaining was performed using the Dako Envision+ Rabbit Peroxidase Detection System (Dako Cytomation, Carpinteria, CA, USA). Antigen retrieval was performed by microwave heating in citrate buffer $(\mathrm{pH} 6.0)$ for 30 min. Peroxidase activity was blocked with $3 \% \mathrm{H}_{2} \mathrm{O}_{2}$-methanol for $10 \mathrm{~min}$ and the sections were incubated with normal goat serum (Dako Cytomation, Carpinteria, CA, USA) for $20 \mathrm{~min}$ to block non-specific antibody binding sites. The sections were incubated with rabbit anti-Gremlin1 antibody (lot\# ab22138, Abcam, Cambridge, UK) for $1 \mathrm{~h}$ at room temperature, followed by incubation with Envision+ anti-rabbit peroxidase for $1 \mathrm{~h}$. For color reactions, sections were incubated with the DAB SubstrateChromogen Solution (Dako Cytomation, Carpinteria, CA, USA) for $10 \mathrm{~min}$. The sections were then counterstained with $0.1 \%$ hematoxylin. Negative controls were created by omitting the addition of the primary antibody.

Gremlin1 expression was scored as positive or negative in all tumors. When more than $1 \%$ of tumor cells were stained for Gremlin1, the immunostaining was considered positive. Using these definitions, two surgical pathologists (NS, AI, and DT) independently reviewed the immunoreactivity in each specimen without knowledge of the clinical and pathological parameters or patient outcome. Inter-observer differences were resolved by consensus review at a double-headed microscope after independent review.

Phenotypic analysis of GC. The GCs were classified into four phenotypes - gastric $(\mathrm{G})$, intestinal (I), gastric intestinal mixed (GI), and Unclassified (N) - as described below. For phenotypic expression analysis of GC, we analyzed the immunohistochemistry as described above, using four antibodies: anti-MUC5AC, antiMUC6, anti-MUC2, and anti-CD10 (all Novocastra, New Castle, UK). GCs in which more than $10 \%$ of the cells in the section expressed at least one gastric epithelial (MUC5AC or MUC6) or intestinal epithelial (MUC2 or CD10) cell markers were classified as $\mathrm{G}$ or I-type cancers, respectively; sections that showed both gastric and intestinal phenotypes were classified as GI-type and those that lacked both gastric and intestinal phenotypes were classified as $\mathrm{N}$-type. The details are described in a previous study (11).

Statistical methods. Correlations between clinicopathological parameters and Gremlin 1 expression were analyzed using Fisher's exact test. Kaplan-Meier survival curves were constructed for both Gremlin1-positive and Gremlin1-negative patients. The differences between the survival curves were tested for statistical significance using log-rank tests. $p$-Values $<0.05$ were considered statistically significant.

\section{Results}

Gremlin1 expression in non-neoplastic gastric mucosa and GC. We examined Gremlin1 expression based on immunohistochemical staining of normal gastric mucosa, intestinal metaplasia, and a series of 159 GC specimens. In non-neoplastic mucosa, Gremlin1 expression was not detected in most areas (Figure 1A and 1B) and was detected only at the bottom of the crypt in a small number of glands located next to cancerous lesions (data not shown). Cells in the lamina propria and muscular mucosa did not show positivity.

While a previous study in colorectal cancer evaluated Gremlin1 expression by combining the percentages of positive tumor cells and mean intensity, CRC cases with scores above 1 showed significantly better prognosis than CRC cases with a score 0 , with no detectable Gremlin 1 staining (9). Therefore, we estimated immunostaining positivity as the presence of at least one cancer cell showing any visible signals. In total, 59 (37\%) of the $159 \mathrm{GC}$ cases were positive for Gremlin1 expression. The staining was granular and/or cytoplasmic (Figure 1C and D), which was consistent with the staining pattern in colorectal cancer (9). The proportion of Gremlin1 positivity ranged from 0 to $50 \%$; most of the Gremlin1positive cases had less than $10 \%$ positive cancer cells.

Correlation between Gremlinl expression and clinicopathological features in GC. The correlations between Gremlin1 expression and clinicopathological characteristics were investigated (Table I). Gremlin1-negative cases showed more advanced T grade ( $p=0.0466$, Fisher's exact test), $\mathrm{N}$ grade $(p=0.0087$, Fisher's exact test), TNM stage ( $p=0.0050$, Fisher's exact test), and tended to have intestinal histology ( $p=0.0226$, Fisher's exact test).

Correlations between Gremlinl expression and mucin phenotype in $G C$. In addition to the Lauren histology-based classification, GC can be subdivided into four phenotypes according to mucin expression(2). Gastric and intestinal markers were detected, including MUC5AC in 78 of 145 (54\%) cases, MUC6 in $16(11 \%)$ cases, MUC2 in $37(25 \%)$ cases, and CD10 in $17(11 \%)$ cases. Only MUC5AC expression was significantly correlated with Gremlin1 expression in GC cases (Table II). We further investigated the association between Gremlin1 expression and mucin phenotype, observing that Gremlin1 expression was more frequently detected in $\mathrm{GC}$ cases with $\mathrm{G}$ types than in cases with other types (Figure 2).

Correlation between Gremlinl expression and survival in GC patients. The relationship between Gremlin 1 expression and survival probability was examined in 159 GC cases. The 5year overall survival rates were $86 \%$ and $58 \%$ for Gremlin1positive and -negative cases, respectively, and the Gremlin1negative GC cases had significantly lower survival probability 

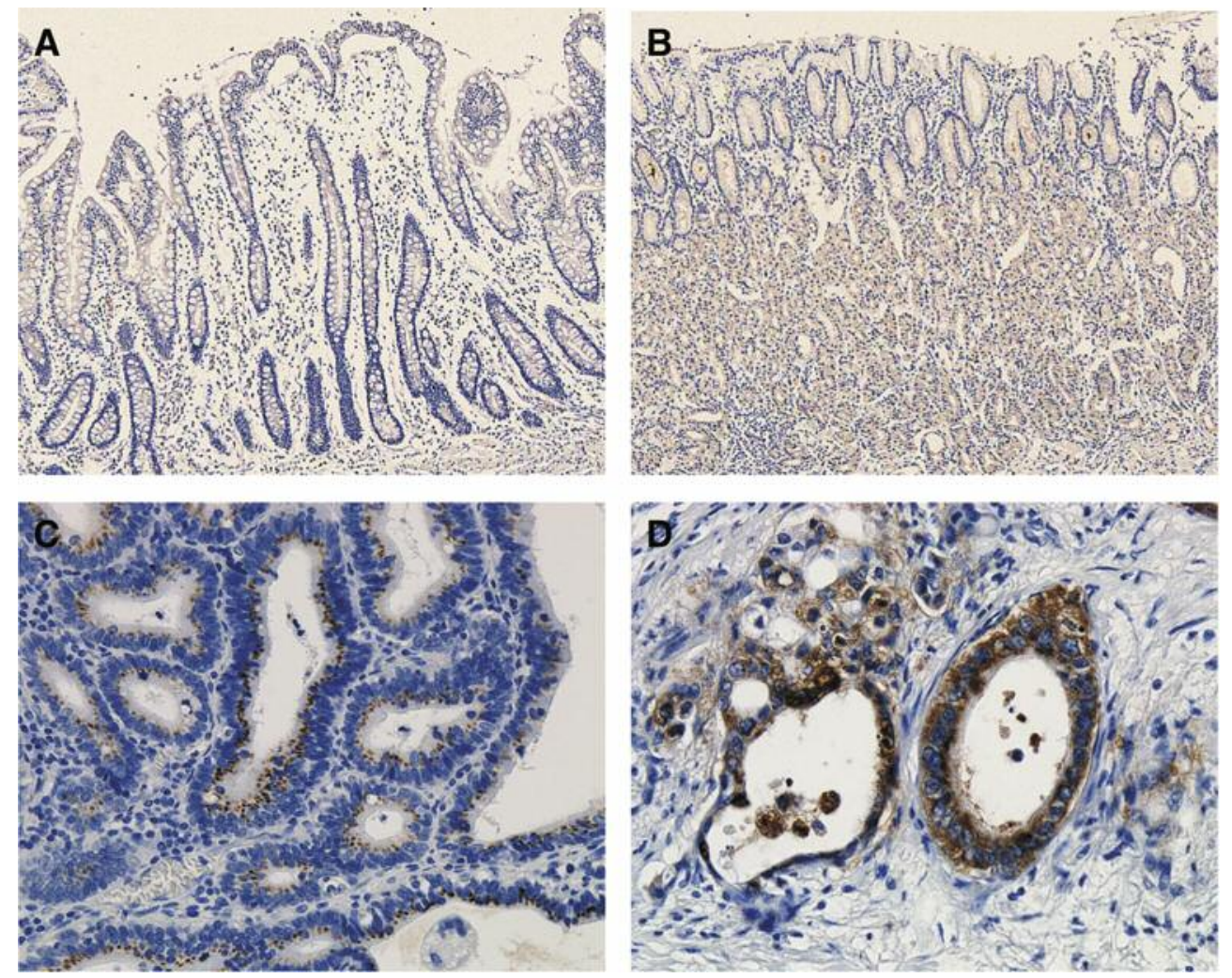

Figure 1. Immunohistochemical analysis of Gremlin1 in non-neoplastic gastric mucosa and gastric cancer (GC). No visible staining was observed in the fundic gland $(A)$ and intestinal metaplasia $(B)$ (Original magnification: $\times 40$ ). Granular $(C)$ and cytoplasmic $(D)$ staining was visible in GC (original magnification: $\times 400$ ).

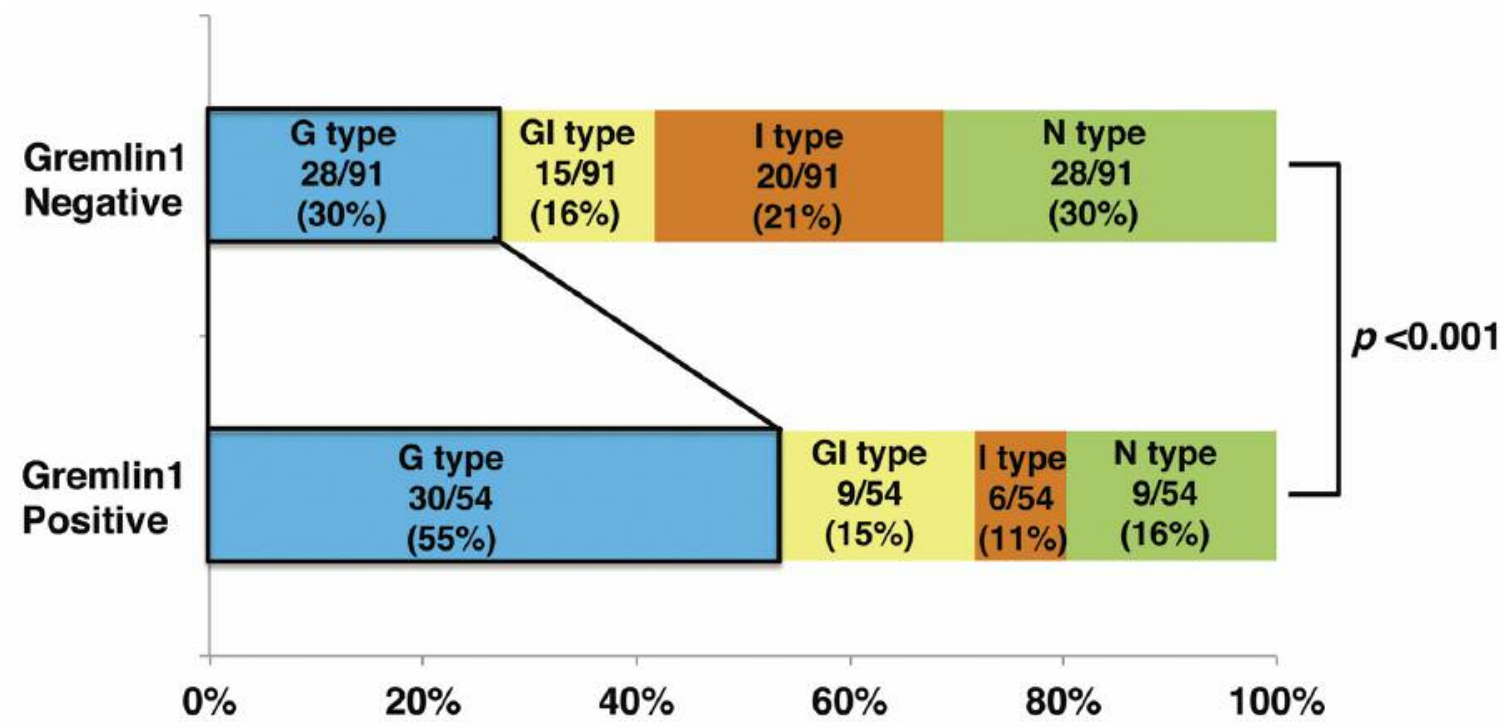

Figure 2. Distribution of gastric (G), intestinal (I), gastric and intestinal mixed (GI), and unclassified (N) phenotypes in Gremlin1-positive gastric cancer $(G C)$ cases. Gremlin1-positive cases were more frequently found in G-type GC than in the other types. p-Values were analyzed using Fisher's exact tests. 
Table I. Clinicopathologic characteristics of 159 GC cases.

\begin{tabular}{|c|c|c|c|c|c|}
\hline & & & \multicolumn{2}{|c|}{ Gremlin 1 expression } & \multirow[b]{2}{*}{$p$-Value* } \\
\hline & & & Negative & Positive & \\
\hline \multirow{2}{*}{$\begin{array}{l}\text { Median age, } \\
\text { years (range) }\end{array}$} & $65(34-87)$ & $<65$ & 53 & 30 & 0.8698 \\
\hline & & $\geq 65$ & 47 & 29 & \\
\hline \multicolumn{6}{|l|}{ Gender $(\%)$} \\
\hline Male & $99(61)$ & Male & 64 & 35 & 0.6128 \\
\hline Female & $60(39)$ & Female & 36 & 24 & \\
\hline \multicolumn{6}{|l|}{$\mathrm{T}$ grade $(\%)$} \\
\hline $\mathrm{T} 1$ & $69(30)$ & $\mathrm{T} 1$ & 37 & 32 & 0.0466 \\
\hline $\mathrm{T} 2$ & $43(26)$ & $\mathrm{T} 2 / 3 / 4$ & 63 & 27 & \\
\hline $\mathrm{T} 3$ & $39(31)$ & & & & \\
\hline $\mathrm{T} 4$ & $8(13)$ & & & & \\
\hline \multicolumn{6}{|l|}{$\mathrm{N}$ grade $(\%)$} \\
\hline No & $86(58)$ & No & 46 & 40 & 0.0087 \\
\hline N1 & $36(58)$ & $\mathrm{N} 1 / 2 / 3$ & 54 & 19 & \\
\hline $\mathrm{N} 2$ & $26(27)$ & & & & \\
\hline N3 & $11(46)$ & & & & \\
\hline \multicolumn{6}{|l|}{ Stage $(\%)$} \\
\hline I & $81(14)$ & I & 42 & 39 & 0.0050 \\
\hline II & $22(21)$ & II/III/IV & 58 & 20 & \\
\hline III & $47(47)$ & & & & \\
\hline IV & $9(18)$ & & & & \\
\hline \multicolumn{6}{|l|}{ Histology $(\%)$} \\
\hline Intestinal & & Intestinal & 42 & 36 & 0.0226 \\
\hline Diffuse & & Diffuse & 58 & 23 & \\
\hline
\end{tabular}

*Fisher's exact test.

than that of the Gremlin 1-positive GC cases ( $p=0.002$; Figure 3A). As Gremlin1 expression was frequently found in G-type GC, we further investigated the association between its expression and patient survival in the 58 G-type GC cases. Kaplan-Meier analysis showed significantly poorer survival in Gremlin1-negative GC cases than that in Gremlin1-positive GC cases ( $p=0.001$; Figure 3B).

In order to evaluate Gremlin1 as a prognostic classifier, the association between its expression and cancer-specific mortality was evaluated in univariate and multivariate Cox proportional hazards analyses. In univariate analysis, $\mathrm{T}$ grade (hazard ratio $[\mathrm{HR}]=14.801 ; 95 \%$ confidence interval $[\mathrm{CI}]=5.408-60.886 ; \quad p=0.001), \quad \mathrm{N}$ grade $\quad(\mathrm{HR}=9.128$; $95 \% \mathrm{CI}=1.267-16.392 ; p<0.001)$, TNM stage $(\mathrm{HR}=8.460$; $95 \% \mathrm{CI}=2.083-11.111 ; \quad p<0.001), \quad$ histological type $(\mathrm{HR}=3.369 ; 95 \% \mathrm{CI}=1.928-6.477 ; p=0.001)$, and Gremlin 1 expression $(\mathrm{HR}=2.734 ; 95 \% \mathrm{CI}=1.132-5.694 ; p=0.003)$ were associated with survival. In multivariate modeling, Gremlin 1 expression $(\mathrm{HR}=3.468 ; 95 \% \mathrm{CI}=1.214-8.776 ; p<0.001)$ was an independent prognostic indicator (Table III). Thus, immunohistochemical examination of Gremlin1 in GC samples reveals the potential of Gremlin1 as a prognostic biomarker for GC.
Table II. Comparison between Gremlin 1 and markers for mucin phenotype in $G C$.

\begin{tabular}{lccc}
\hline & \multicolumn{2}{c}{ Gremlin1 expression } & \\
\cline { 2 - 3 } & Negative & Positive & $p$-Value* \\
\hline Gastric markers & & & \\
MUC5AC & & & \\
$\quad$ Positive & 30 & 48 & $<0.001$ \\
$\quad$ Negative & 56 & 11 & \\
MUC6 & 7 & & \\
Positive & 79 & 50 & \\
$\quad$ Negative & & & \\
Intestinal markers & & & \\
MUC2 & & & \\
$\quad$ Positive & 21 & 16 & 0.8465 \\
$\quad$ Negative & 65 & 43 & \\
CD10 & & & \\
Positive & 10 & 7 & 0.9882 \\
$\quad$ Negative & 76 & 52 & \\
\hline
\end{tabular}

*Fisher's exact test.

\section{Discussion}

The results of the present study demonstrate the clinical significance of Gremlin1 expression in GC, including evidence that Gremlin1-negative GC cases are more likely to be advanced cancer and have a poorer prognosis, and that Gremlin1 expression is an independent prognostic factor in GC. BMPs have been established as functional regulators in the maintenance of homeostasis and sequential metaplastic and dysplastic changes in normal stomach tissue (12). In the light of the functional role of Gremlin1, an antagonist of several BMPs, Gremlin1 is presumably involved in biological processes in normal stomach and/or GC tissue, although no studies have described the functional roles of Gremlin1 in GC tissue.

A previous study reported Gremlin 1 to be preferentially expressed in CRC cases with serrated morphology (9). Colorectal serrated tumors often display gastric features (13) and CRCs with serrated morphology are often categorized as "hypermutated" cases that are frequently accompanied by microsatellite instability (MSI), $\mathrm{CpG}$ island methylator phenotype (CIMP), and several mutations in the genes related to the TGF- $\beta$ signal pathway, including $A C V R 2$ and TGFBR2 (14). In the present study, Gremlin1 expression was significantly associated with MUC5AC expression and was often detected in G-type GC. G-type GC may represent MSI-H, a frequent methylation in the promoter region of tumor suppressor genes and less frequent p53 mutations (2). Recent evidence suggest that GCs in the "MSI-group", 
A

All GC cases $(n=159)$

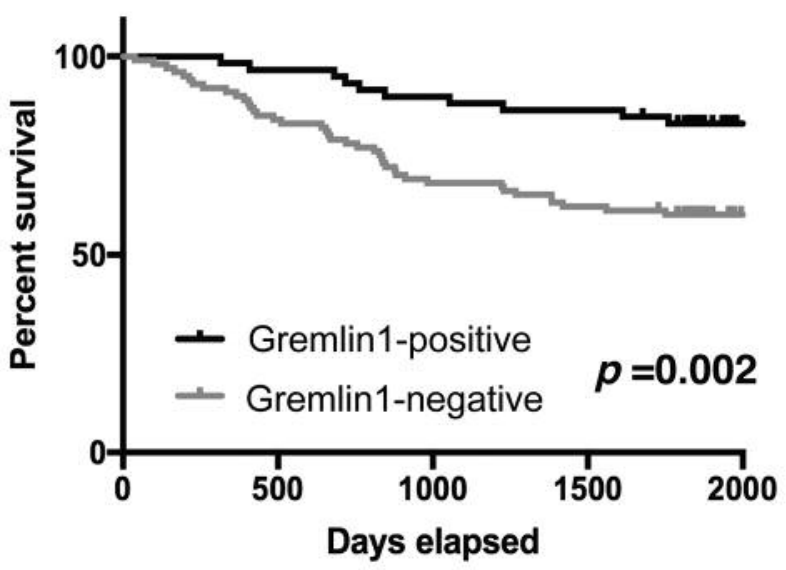

B

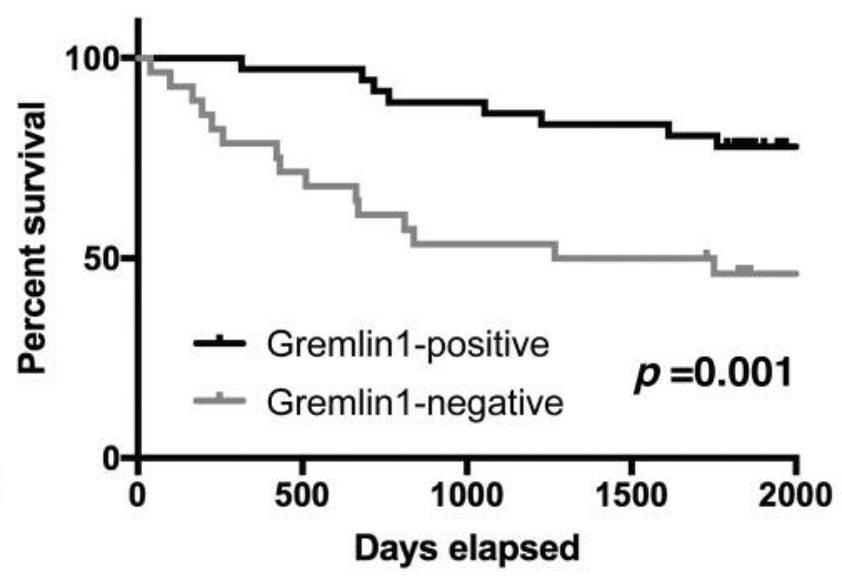

Figure 3. Survival of patients with Gremlin1-positive and-negative gastric cancer (GC). (A) Kaplan-Meier curves of patients with Gremlin1-positive and-negative GC in a series of 159 GC cases. (B) Kaplan-Meier curves of patients with Gremlin1-positive and -negative GC in 59 G-type GC cases.

Table III. Univariate and multivariate analysis of factors influencing survival in 159 GC cases.

\begin{tabular}{|c|c|c|c|c|c|c|}
\hline \multirow[b]{2}{*}{ Parameter } & \multicolumn{3}{|c|}{ Univariate analysis } & \multicolumn{3}{|c|}{ Multivariate analysis } \\
\hline & $\mathrm{HR}^{\mathrm{a}}$ & $95 \% \mathrm{CI}^{\mathrm{b}}$ & $p$-Value & HR & $95 \% \mathrm{CI}$ & $p$-Value \\
\hline \multicolumn{7}{|l|}{ Gender } \\
\hline Female & Ref & & & & & \\
\hline Male & 1.131 & $0.685-2.152$ & 0.6894 & & & \\
\hline \multicolumn{7}{|l|}{ Age } \\
\hline$<65$ y.o & Ref & & & & & \\
\hline$>65$ y.o & 1.122 & $0.442-1.483$ & 0.8593 & & & \\
\hline \multicolumn{7}{|l|}{$\mathrm{T}$ grade } \\
\hline pT1 & Ref & & & Ref & & \\
\hline $\mathrm{pT} 2 / \mathrm{T} 3 / \mathrm{T} 4$ & 14.801 & $5.408-60.886$ & 0.001 & 1.322 & $0.248-9.085$ & 0.774 \\
\hline \multicolumn{7}{|l|}{$\mathrm{N}$ grade } \\
\hline pNO & Ref & & & Ref & & \\
\hline $\mathrm{pN} 1 / \mathrm{N} 2 / \mathrm{N} 3$ & 9.128 & $1.267-16.392$ & $<0.001$ & 4.979 & $0.928-89.438$ & 0.0325 \\
\hline \multicolumn{7}{|l|}{ TNM stage } \\
\hline pStage I & Ref & & & Ref & & \\
\hline pStage II/III/IV & 8.460 & $2.083-11.111$ & $<0.001$ & 34.579 & $6.293-222.27$ & $<0.001$ \\
\hline \multicolumn{7}{|l|}{ Histrogical type } \\
\hline Intestinal & Ref & & & Ref & & \\
\hline Diffuse & 3.369 & $1.928-6.477$ & 0.001 & 0.652 & $0.333-1.381$ & 0.267 \\
\hline \multicolumn{7}{|c|}{ Gremlin1 expression } \\
\hline Positive & Ref & & & Ref & & \\
\hline Negative & 2.734 & $1.132-5.694$ & 0.003 & 3.468 & $1.214-8.776$ & 0.017 \\
\hline
\end{tabular}

aHR: Hazard ratio; ${ }^{b} \mathrm{CI}$ : confidence interval.

defined as TCGA based on data from the comprehensive characterization of GC by whole-genome sequencing, frequently have mutations in activin type II receptor (ACVR2) and transforming growth factor beta receptor 2
(TGFBR2) (15). Taken together, the previous data and our own indicate that Gremlin 1 expression is probably disturbed in both CRC and GC cells with similar genetic and phenotypic backgrounds. Only one paper has described the 
details of a proposed mechanism concerning Gremlin1 overexpression, which is confirmed by a rare hereditary polyposis syndrome; human hereditary mixed polyposis syndrome (16). Further in-depth studies are still needed to characterize the mechanism underlying the dysregulation of Gremlin1 expression, which may also reveal the fundamental mechanisms commonly involved in GC and CRC progression.

Another important issue is that the antibody used in the present study does not detect Gremlin 1 expression in cells from the stromal components. Evidence indicates that Gremlin1 expression in intestinal pericryptal fibroblasts and smooth muscle cells is present in an increasing gradient alongside the crypts, which may contribute to the maintenance of the intestinal stem cell niche in the colonic basal crypt region (5). In situ hybridization for GREMI has identified the expression of GREM1 in both epithelial and stromal cells in the colon and GREM1-negative CRCs in the stromal cells showed significantly more advanced TNM stage and worse prognosis compared to those of GREM1positive CRCs, whereas there were no significant correlations among epithelial GREM1 positivity and these factors (4). In terms of the difference in the stromal tissue of the gastrointestinal tract, there is a definitive signature in both the stomach and colon. One of the biggest differences is BARX1, which could interact with CDX2, an essential transcription factor of the colon epithelium (17). Thus, stromal Gremlin1 expression in normal stomach and GC tissue could be regulated in a different manner than that in the normal colon and CRC. Additional studies are needed to measure the stromal Gremlin1 expression in normal stomach and GC, which could further verify the importance of Gremlin1 expression in these tissues.

In summary, we verified the staining property of Gremlin 1 in normal, metaplastic, and cancerous tissues of the stomach. The expression status of epithelial Gremlin1 was significantly associated with tumor progression and was also an independent marker for poor survival in patients with GC. Although the detailed function of Gremlin1 in GC remains unclear, these data highlight its potential as a prognostic classifier in patients with GC.

\section{Acknowledgements}

The Authors thank Mr. Shinichi Norimura for his technical assistance. This work was carried out with kind cooperation from the Research Center for Molecular Medicine of the Faculty of Medicine of Hiroshima University. The Auhtors would also like to thank the Analysis Center of Life Science of Hiroshima University for the use of their facilities. This work was supported by Grants-in-Aid for Scientific Research (JP15H04713, JP16K08691, JP16H06999) and Challenging Exploratory Research (26670175, JP16K15247) from the Japan Society for the Promotion of Science.

\section{References}

1 Yasui W, Oue N, Sentani K, Sakamoto N and Motoshita J: Transcriptome dissection of gastric cancer: identification of novel diagnostic and therapeutic targets from pathology specimens. Pathol Int 59: 121-136, 2009.

2 Oue N, Sentani K, Sakamoto N and Yasui W: Clinicopathologic and molecular characteristics of gastric cancer showing gastric and intestinal mucin phenotype. Cancer Sci 106: 951-958, 2015.

3 Wordinger RJ, Zode G and Clark AF: Focus on molecules: gremlin. Exp Eye Res 87: 78-79, 2008.

4 Bragdon B, Moseychuk O, Saldanha S, King D, Julian J and Nohe A: Bone morphogenetic proteins: a critical review. Cell Signal 23: 609-620, 2011.

5 Kosinski C, Li VS, Chan AS, Zhang J, Ho C, Tsui WY, Chan TL, Mifflin RC, Powell DW, Yuen ST, Leung SY and Chen X: Gene expression patterns of human colon tops and basal crypts and BMP antagonists as intestinal stem cell niche factors. PNAS 104: 15418-15423, 2007.

6 Namkoong H, Shin SM, Kim HK, Ha SA, Cho GW, Hur SY, Kim TE and Kim JW: The bone morphogenetic protein antagonist gremlin 1 is overexpressed in human cancers and interacts with YWHAH protein. BMC Cancer 6: 74, 2006.

7 Laurila R, Parkkila S, Isola J, Kallioniemi A and Alarmo EL: The expression patterns of gremlin 1 and noggin in normal adult and tumor tissues. Int lin Xp Athol 6: 1400-1408, 2013.

8 Davis H, Irshad S, Bansal M, Rafferty H, Boitsova T, Bardella C, Jaeger E, Lewis A, Freeman-Mills L, Giner FC, RodenasCuadrado P, Mallappa S, Clark S, Thomas H, Jeffery R, Poulsom R, Rodriguez-Justo M, Novelli M, Chetty R, Silver A, Sansom OJ, Greten FR, Wang LM, East JE, Tomlinson I and Leedham SJ: Aberrant epithelial GREM1 expression initiates colonic tumorigenesis from cells outside the stem cell niche. Nat Med 21: 62-70, 2015.

9 Pelli A, Vayrynen JP, Klintrup K, Makela J, Makinen MJ, Tuomisto A and Karttunen TJ: Gremlin1 expression associates with serrated pathway and favourable prognosis in colorectal cancer. Histopathology 69: 831-838, 2016.

10 Lauren P: The two histological main types of gastric carcinoma: diffuse and so-called intestinal-type carcinoma. an attempt at a histo-clinical classification. Acta icrobiol Scand 64: 31-49, 1965.

11 Sakamoto N, Oue N, Sentani K, Anami K, Uraoka N, Naito Y, Oo HZ, Hinoi T, Ohdan H, Yanagihara K, Aoyagi K, Sasaki $\mathrm{H}$ and Yasui W: Liver-intestine cadherin induction by epidermal growth factor receptor is associated with intestinal differentiation of gastric cancer. Cancer Sci 103: 1744-1750, 2012.

12 Todisco A: Regulation of gastric metaplasia, dysplasia and neoplasia by bone morphogenetic protein signaling. Cell Mol Gastroenterol Hepatol 3: 339-347, 2017.

13 Sakamoto N, Feng Y, Stolfi C, Kurosu Y, Green M, Lin J, Green ME, Sentani K, Yasui W, McMahon M, Hardiman KM, Spence JR, Horita N, Greenson JK, Kuick R, Cho KR and Fearon ER: BRAF(V600E) cooperates with CDX2 inactivation to promote serrated colorectal tumorigenesis. Elife 6: e20331, 2017.

14 Cancer Genome Atlas N: Comprehensive molecular characterization of human colon and rectal cancer. Nature 487 : 330-337, 2012. 
15 Wang K, Yuen ST, Xu J, Lee SP, Yan HH, Shi ST, Siu HC, Deng S, Chu KM, Law S, Chan KH, Chan AS, Tsui WY, Ho SL, Chan AK, Man JL, Foglizzo V, Ng MK, Chan AS, Ching YP, Cheng GH, Xie T, Fernandez J, Li VS, Clevers H, Rejto PA, Mao M and Leung SY: Whole-genome sequencing and comprehensive molecular profiling identify new driver mutations in gastric cancer. Nat Genet 46: 573-582, 2014.

16 Jaeger E, Leedham S, Lewis A, Segditsas S, Becker M, Cuadrado PR, Davis H, Kaur K, Heinimann K, Howarth K, Collaboration H, East J, Taylor J, Thomas $\mathrm{H}$ and Tomlinson I: Hereditary mixed polyposis syndrome is caused by a $40-\mathrm{kb}$ upstream duplication that leads to increased and ectopic expression of the BMP antagonist GREM1. Nat Genet 44: 699703,2012
17 Stringer EJ, Duluc I, Saandi T, Davidson I, Bialecka M, Sato T, Barker N, Clevers H, Pritchard CA, Winton DJ, Wright NA, Freund JN, Deschamps J and Beck F: Cdx2 determines the fate of postnatal intestinal endoderm. Development 139: 465-474, 2012.

Received December 11, 2017

Revised December 25, 2017

Accepted December 29, 2017 